\title{
Long-Distance Dermatology: Lessons From an Interview on Remote Practice During a Pandemic and Beyond
}

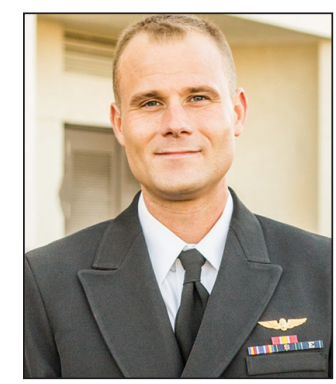

\author{
W. Hugh Lyford, MD
}

\section{RESIDENT PEARL}

- One result of the COVID-19 pandemic is the aggressive adoption of teledermatology across the United States. Graduating residents should be preparing for a scope of practice that incorporates teledermatology.

The COVID-19 pandemic resulted in profound changes to most facets of medical practice. The field of dermatology has adapted by rapidly incorporating teledermatology as a means of evaluating, treating, and staying connected with our patients. Broader dermatology access, convenience to patients, and value to payers are benefits to this rapidly evolving practice model and suggest that teledermatology will be a part of day-to-day practice even as the worst of the pandemic is behind us. This interview provides one recent dermatology resident graduate's experiences incorporating teledermatology into his practice model and provides advice for future residents on preparing to do the same.

Cutis. 2021:107:E37-E39.

F or the US health care system, the year 2020 was one of great change as well as extreme pain and hardship: some physical, but much emotional and financial. Dermatologists nationwide have not been sheltered from the winds of change. Yet as with most great challenges, one also can discern great change for the better if you look for it. One area of major growth in the wake of the COVID-19 pandemic is the expansion of telehealth, specifically teledermatology.

Prior to the pandemic, teledermatology was in a phase of modest expansion. ${ }^{1}$ Since the start of the pandemic, however, the adoption of telemedicine services in the United States has been beyond exponential. Before the pandemic, an estimated 15,000 Medicare recipients received telehealth services on a weekly basis. Yet by the end of April 2020, only 3 months after the first reported case of COVID-19 in the United States, nearly 1.3 million Medicare beneficiaries were utilizing telehealth services on a weekly basis. ${ }^{2}$ The Centers for Medicare \& Medicaid Services has recognized the vast increase in need and responded with the addition of 144 new telehealth services covered by Medicare in the last year. In December 2020, the Centers for Medicare \& Medicaid Services moved to make many of the previously provisional policies permanent, expanding long-term coverage for telehealth services, ${ }^{2}$ and use of teledermatology has expanded in parallel. Although the impetus for this change was simple necessity, the benefits of expanded teledermatology are likely to drive its continued incorporation into our daily practices.

From the Department of Dermatology, Naval Medical Center, San Diego, California.

The author reports no conflict of interest.

The views expressed in this article reflect the results of research conducted by the author and do not necessarily reflect the official policy or position of the Department of the Navy, Department of Defense, or the United States Government.

Correspondence: W. Hugh Lyford, MD, Naval Medical Center, Department of Dermatology, 34800 Bob Wilson Dr, San Diego, CA 92134

(willis.h.lyford.mil@mail.mil).

doi:10.12788/cutis.0242 
Kevin Wright, MD, is a staff dermatologist at the Naval Medical Center San Diego (San Diego, California) and an Associate Professor of Dermatology at the Uniformed Services University of the Health Sciences (Bethesda, Maryland). In this interview, we discussed his experience incorporating a teledermatology component into his postresidency practice, the pros and cons of teledermatology practice, and ways that residents can prepare for a future in teledermatology.

\section{Would you start by briefly describing your work model now?}

My primary job is a Monday-through-Friday classic dermatology clinic job. On the weekends or days off, I see asynchronous and synchronous teledermatology through a specialized platform. On weekends, I tend to see anywhere between 20 and 40 patients in about a 6-hour period with breaks in between.

\section{What does a typical "weekend" day of work look like?}

In general, I'll wake up early before my family and spend maybe an hour working. Oftentimes, that will be in my truck parked down by the beach, where I will go for a run or surf before logging on. If I have 40 visits scheduled that day, I can spend a few hours, message most of them, clarify some aspects of the visit, then go and have breakfast with my family before logging back on and completing the encounters.

\section{Is most of your interaction with patients asynchronous, messaging back and forth to take history?}

A few states require a phone call, so those are synchronous, and every Medicaid patient requires a video call. I do synchronous visits with all of my isotretinoin patients at first. It's a mixed bag, but a lot of my visits are done entirely asynchronously.

\section{What attracted you to this model?}

During residency, I always felt that many of the ways we saw patients seemed extraordinarily inefficient. My best example of this is isotretinoin follow-ups. Before this year, most of my colleagues were uncomfortable with virtual isotretinoin follow-ups or thought it was a ridiculous idea. Frankly, I never shared this sentiment. Once I had my own board certification, I knew I was going to pursue teledermatology, because seeing kids take a half day off of school to come in for a 10 -minute isotretinoin appointment (that's mainly just a conversation about sports) just didn't make sense to me. So I knew I wanted to pursue this idea, I just didn't know exactly how. One day I was approached by a close friend and mentor of mine who had just purchased a teledermatology platform. She asked me if I would like to moonlight once I graduated and I jumped at the opportunity.

\section{What steps did you take prior to graduating to help prepare you to practice teledermatology?} The most important thing I did-and the most important thing I think for third-year residents to do-is to set myself up for success by starting the US Drug Enforcement Administration (DEA) licensure and certification process. Once you have a DEA number, you can apply for Medicare and Medicaid. The nice thing about Medicare is you can start billing immediately after you apply, which is important. The reimbursement isn't as high, but they pay faster, which allows you to start seeing patients through teledermatology right away. In a pinch, you could see all Medicare patients and make a living until you've completed the rest of the process. Once you have a Medicare and Medicaid number, you can apply for credentialing through private payers. However, the Medicare process takes 3 months, and private-payers credentialing takes about 90 days as well. That's a lot of time! Before finishing residency, I recommend you make sure you have an unrestricted DEA license and you apply for Medicare/Medicaid credentials. Then, when you're looking at future employment, you can start getting state licenses almost immediately in whatever states you anticipate needing them.

\section{What are the top 3 benefits of incorporating teledermatology into your practice?}

Accessibility is one huge benefit. If you're practicing in a rural area, you're basically giving [patients] back their time. Teledermatology takes patients much less time, and they get the same level of care. That's a big selling point. Your patients will be very happy and loyal because of that.

The other thing I never would have foreseen before starting teledermatology is the amazing followup you can get. I think many dermatology residents will agree that there are those patients where you think, "Wow, I wish I could see them back. I wonder how they did," but you never see them again. That's not the case with teledermatology. I have a running list of all my interesting cases, and I'll just shoot them a message 2 or 4 weeks later and at their convenience, they can submit a quick photo. I get that excellent feedback, and that's huge to me for my own personal education and growth.

The third would be experience. I have 24 state medical licenses, and I see patients of all varieties: all socioeconomic backgrounds and skin types and many with severe skin conditions never managed before by a specialist. That, frankly, has increased my comfort level for seeing patients of all types. It forces me to expand my utilization of certain therapies because some people can't afford 95\% of medications we prescribe commonly. I find that challenge very rewarding. It's something I'm not sure you can achieve by just practicing within your bubble. Inevitably you are going to see a certain type of patient that your hospital or practice attracts by merit of its geography or catchment area. Teledermatology allows you to see the full spectrum of dermatology. 


\section{What are the biggest cons to incorporating teledermatology into your practice?}

To start off, some patients have boundary issues. Every 200 patients or so, I'll have someone who submits a visit at 11:30 PM, and then at 1:00 or 2:00 AM they're asking, "Why am I not being seen, what's going on?"Maintaining patient boundaries becomes exponentially more difficult. In some respects, you are now expected to be available 24/7 because some people have unreasonable expectations. That is one of the most difficult aspects of practicing the way I do.

The second is reimbursement. In other practice models I can bill more in half the time by seeing a patient in person, doing a skin screening and a few biopsies. I believe there's always a role for teledermatology in any practice, but ultimately dermatologists are pragmatic people who need to be smart about time management. At some point, it becomes difficult to pay the bills if reimbursement is lacking. That's one of the bigger downsides to teledermatology. We still need to figure out how to reimburse to incentivize what's best for the patient.

\section{Could you talk more about the effect on work-life balance?}

I think the things that make teledermatology appealing are the same things that could end up disrupting your work-life balance. On the positive side, you can vacation in Hawaii, work for 2 hours each morning, and pay for the whole thing. That's very appealing to me! The downside is that there are always patients in the queue. In some sense, your waiting room is always half-full, $24 / 7$. Mentally, you have to become comfortable with that, and you have to develop boundaries. I have very specific times I do teledermatology and then I log off. This helps me establish boundaries and creates balance.

\section{You touched on it earlier regarding isotretinoin visits, but what other facets of practice do you think are particularly well-suited to teledermatology?}

There are a few that I've incorporated into my practice quite aggressively. Almost all acne is going to go to a teledermatology visit. That's in large part due to payer parity. For the most part, you make the same doing an acne visit online as you will doing it in person. Your patients will be getting the same level of care, better follow-up, and you'll make the same amount of money. Another thing I do as a patient courtesy is wound checks postsurgery or post-Mohs [micrographic surgery]. There is a huge benefit there to seeing your patients because you can identify infections early, answer simple questions, and reduce inperson clinic visits. That's a win.

\section{What are visit types you feel are not well-suited to teledermatology or that you approach with more caution?}

This will be different for everyone to some degree. I think practitioners need to be alert and use their best judgement when approaching any new patient or new concern. Pigmented lesions certainly give me pause. Although the technology is getting better every day, I believe there is still a gap between seeing a photo of a lesion and seeing a pigmented lesion in person, being able to get up close and examine it dermoscopically. Teledermoscopy, however, is an emerging business model as well, and it will be interesting to see what role this can play as it gets incorporated.

\section{You mentioned having medical licenses in sev- eral states. Can you describe the process you went through to obtain these licenses?}

It's a painful process. I started realizing this was something I wanted to incorporate after residency, so I started looking into applying for medical licenses early. Teledermatology companies often will reimburse you and help you to get licenses. I was lucky enough to get assistance, which was essential because it is an onerous process. If you can work that into your contract during negotiations that would be ideal. Not everyone will be as lucky as I was, though. If that doesn't pertain to you, pick a few states that have larger populations around you, where you know that they have a lot of need and start applying there. Be aware that medical licensure takes about 6 months. Having this started around mid-third year is important.

Employers want someone they can use right away, so I found it very beneficial to approach an employer and be able to explain to them tangibly where you are in the process. For example, "I've got my DEA license, Medicare, Medicaid number, and I have licensure in your state and all the surrounding states." You then have a leg to stand on with your negotiating. If you do the legwork and can then negotiate a higher percentage, you'll make up the licensure fees in a half day of work. It's an investment toward your professional career.

\section{Any final thoughts?}

I think that insurers are very interested in teledermatology because there's a potential for huge cost savings. As the dust settles with COVID-19 and we see how telemedicine has changed medicine in general, I really think that payers are going to be more aggressive about requiring teledermatology from their dermatologists. I think residents need to anticipate that teledermatology will be some part of their practice in the future and should start planning now to be prepared for this brave new world going forward.

\section{REFERENCES}

1. Yim KM, Florek AG, Oh $\mathrm{DH}$, et al. Teledermatology in the United States: an update in a dynamic era. Telemed J E Health. 2018;24:691-697.

2. Shatzkes MM, Borha EL. Permanent expansion of Medicare telehealth services. The National Law Review website. Published December 7, 2020. Accessed April 13, 2021. https://www.natlawreview.com/article/ permanent-expansion-medicare-telehealth-services 